\title{
THE SOBOLEV EMBEDDINGS ARE USUALLY SHARP
}

\author{
A. FRAYSSE AND S. JAFFARD
}

Received 10 November 2003

Let $x_{0} \in \mathbb{R}^{d}$; we study the Hölder regularity at $x_{0}$ of a generic function of the Sobolev space $L^{p, s}\left(\mathbb{R}^{d}\right)$ and of the Besov space $B_{p}^{s, q}\left(\mathbb{R}^{d}\right)$ for $s-d / p>0$. The setting for genericity is supplied here by HP-residual sets.

\section{Introduction and statement of results}

Let $s \in \mathbb{R}$ and $p \geq 1$; the Sobolev space $L^{p, s}\left(\mathbb{R}^{d}\right)$ is the space of tempered distributions $f$ such that $(\operatorname{Id}-\Delta)^{s / 2} f \in L^{p}$, where $(\operatorname{Id}-\Delta)^{s / 2}$ is the Fourier multiplier by $\left(1+|\xi|^{2}\right)^{s / 2}$. If $s>d / p$, then $L^{p, s}$ is composed of continuous functions; more precisely, the Sobolev embeddings state that $L^{p, s} \hookrightarrow C^{s-d / p}$, see [24, Chapter 11]. In order to state in which sense this embedding is sharp, we need to recall the notion of pointwise Hölder exponent.

Definition 1.1. Let $x_{0}$ be a given point of $\mathbb{R}^{d}$; let $\alpha \geq 0$ and $C>0$; a function $f: \mathbb{R}^{d} \rightarrow \mathbb{R}$ is $(C, \alpha)$-smooth at $x_{0}$ if there exists a polynomial $P$ of degree less than $[\alpha]$ such that, if $\left|x-x_{0}\right| \leq 1$, then

$$
\left|f(x)-P\left(x-x_{0}\right)\right| \leq C\left|x-x_{0}\right|^{\alpha}
$$

The function $f$ belongs to $C^{\alpha}\left(x_{0}\right)$ if there exists a constant $C>0$ such that $f$ is $(C, \alpha)$ smooth at $x_{0}$. The Hölder exponent of $f$ at $x_{0}$ is

$$
h_{f}\left(x_{0}\right)=\sup \left\{\alpha: f \in C^{\alpha}\left(x_{0}\right)\right\} \text {. }
$$

If $s>d / p$, then for any $x_{0} \in \mathbb{R}^{d}$, there exist functions $f$ with the following properties:

$$
\begin{gathered}
f \in L^{p, s}, \\
h_{f}\left(x_{0}\right)=s-\frac{d}{p}
\end{gathered}
$$

(see the appendix); because of the Sobolev embeddings, the Hölder exponent $s-d / p$ is the smallest we can expect for functions in $L^{p, s}$. One may wonder if such examples are exceptional or if, on the contrary, "most" functions of $L^{p, s}$ satisfy ( $\mathscr{H}$ ). In order to 
state this problem with some precision, we first recall and compare several notions of genericity that have been introduced in the past.

We assume in the following that $E$ is a complete metric vector space (in this paper, we will only consider the cases of Sobolev and Besov spaces). The first notion of genericity, introduced by René-Louis Baire in 1899, is supplied by Baire's categories: a property holds quasi-everywhere in $E$ if it holds at least on a countable intersection of dense open subsets of $E$, that is, on a set of first Baire category. One of the first spectacular uses of this notion in the context of Hölder regularity was the proof by Banach and Mazurkiewicz in 1931 that quasi-every continuous function is nowhere differentiable, see $[2,17]$. This result was immediately improved by Auerbach and Banach who proved that the Hölder exponent of quasi-every continuous function vanishes everywhere, see [1]. Baire results are of a topological nature; an alternative is supplied by measure-type results: if $E$ is endowed with a measure $\mu$, a result holds almost everywhere if it holds outside a set of measure 0 . The problem is that this notion is by no means intrinsic, but is highly dependent on the measure $\mu$ which is chosen. A remarkable way to solve this problem and recover a canonical notion of almost everywhere was discovered by Christensen in 1972, and is defined as follows, see $[3,9]$.

Definition 1.2. Let $E$ be a metric Banach space. A Borel subset $A$ of $E$ is a Haar null set if there exists a compactly supported probability measure $\mu$ on $E$ such that

$$
\forall x \in E, \quad \mu(A+x)=0 .
$$

A set is prevalent if its complement is Haar null.

In 1994, Hunt showed that the result of Banach and Auerbach also holds in the setting of prevalence: the Hölder exponent of a prevalent set of functions of $\mathscr{C}(\mathbb{R})$ vanishes everywhere, see [8]. Baire-type results concerning the pointwise regularity of functions in either Sobolev or Besov spaces were investigated in [13] (and in [14] for the critical case $s=d / p$ ) and their counterpart for prevalence in [6].

Baire categories and prevalence share the properties that are expected to hold for any "reasonable" notion of genericity in a topological vector space: generic sets are dense and they are also stable by translation, dilation, and countable intersection. However, these two notions of genericity usually differ widely; for instance, if $E=\mathbb{R}^{d}$, prevalence coincides with "Lebesgue almost everywhere," and there exist subsets of $\mathbb{R}^{d}$ which are generic in the sense of Baire, but have vanishing Lebesgue measure (see [23] for much stronger results of this type in infinite-dimensional spaces). However we just saw that, in the space $\mathscr{C}(\mathbb{R})$, functions whose Hölder exponent vanishes everywhere are generic in both settings. This example is by no means an isolated case and, therefore, it is natural to look for a stronger notion of genericity that would imply both quasi-everywhere and prevalent results and would thus be a proper setting for such properties. (A notion of genericity is stronger than another if the collection of "null sets" that it yields is smaller.) Such a notion was discovered by Kolár in 2001, and is defined as follows, see [15].

Definition 1.3. Let $A$ be a subset of a Banach space $E$ and $c \in(0,1] ; A$ has the property $\operatorname{HP}_{(c)}$ if for every $c^{\prime} \in(0, c)$ and $r>0$, there exist $K>0$ and a sequence of balls $\left\{B_{i}\right\}_{i \in \mathbb{N}}$, 
where $B_{i}=B\left(y_{i}, c^{\prime} r\right)$ and $\left\|y_{i}\right\| \leq r$, such that

$$
\forall x \in E, \quad \operatorname{card}\left\{i \in \mathbb{N}:\left(x+B_{i}\right) \cap A \neq \varnothing\right\} \leq K .
$$

The set $A$ is said to be HP-small if there is $\left(c_{n}\right) \in(0,1]$ such that $A$ is a countable union of sets $A_{n}$ with property $\mathrm{HP}_{\left(c_{n}\right)}$. The complement of an HP-small set is called an HP-residual set.

Kolár proved in [15] that every HP-small set is both Haar null and of first Baire category; furthermore, HP-residual sets share the previously mentioned properties of invariance by translation, dilation, and countable intersection. This new notion is much more demanding than Baire or prevalence genericity; this can already be seen in $\mathbb{R}^{d}$ where the only HP-small set is the empty set. The situation is not so drastic in infinite-dimensional spaces: for instance, in that case, compact sets are HP-small sets, see [15]. We will prove the following result.

Theorem 1.4. Let $p>1, s>d / p$, and let $x_{0} \in \mathbb{R}^{d}$ be fixed. The functions of $L^{p, s}\left(\mathbb{R}^{d}\right)$ having the pointwise Hölder exponent

$$
h_{f}\left(x_{0}\right)=s-\frac{d}{p}
$$

form an HP-residual set of $L^{p, s}$.

Remarks 1.5. (i) We will recall in Section 4 the notion of Gaussian null set which supplies a notion of genericity stronger than prevalence and we will show that it is not a right setting in order to obtain the conclusion of Theorem 1.4.

(ii) In [15], Kolár proved that, in the space of continuous functions, the functions having a Hölder exponent which vanishes everywhere form an HP-residual set.

(iii) For an arbitrary function $f \in L^{p, s},(1.5)$ can hold at most on a set of points $x_{0}$ of dimension 0 ; another indication that this situation is exceptional is shown by the following result: any function of $L^{p, s}$ has Hölder exponent almost everywhere greater than or equal to $s$; both results will be precisely recalled in the appendix.

(iv) If $0 \leq s<d / p$, then it is proved in [6] that almost every function of $L^{p, s}\left(\mathbb{R}^{d}\right)$ (in the sense of prevalence) is nowhere locally bounded; therefore, in this case, one can expect that any function $f$ of an HP-residual set of $L^{p, s}$ is not bounded at $x_{0}$, that is, satisfies

$$
\forall \phi \in \mathscr{D}\left(\mathbb{R}^{d}\right), \quad \phi\left(x_{0}\right) \neq 0 \Longrightarrow f \phi \notin L^{\infty}\left(\mathbb{R}^{d}\right) .
$$

\section{Wavelet expansions and Besov spaces}

In order to prove Theorem 1.4, we first need to recall the definition and the main properties of wavelet expansions, see $[5,7,18,19,20]$. An $N$-smooth wavelet basis is composed of $2^{d}-1$ wavelets $\psi^{(i)}$ which belong to $C^{N}$ and satisfy the following properties.

(i) For all $i$, for all $\alpha$ such that $|\alpha| \leq N, \partial^{\alpha} \psi^{(i)}$ has fast decay.

(ii) The set of functions $2^{d j / 2} \psi^{(i)}\left(2^{j} x-k\right), j \in \mathbb{Z}, k \in \mathbb{Z}^{d}, i \in\left\{1, \ldots, 2^{d}-1\right\}$ is an orthonormal basis of $L^{2}\left(\mathbb{R}^{d}\right)$ and is an unconditional basis of the Sobolev spaces $L^{p, s}$ for $p \in(1, \infty),|s|<N$, see $[18,19,20]$; furthermore, it is an unconditional basis of the Besov 
spaces $B_{p}^{s, q}\left(\mathbb{R}^{d}\right)$ for $p \in(0, \infty), q \in(0, \infty)$, and $|s|<N$, see [7, Theorem 7.20]. (Note that $L^{1, s}$ and $B_{p}^{s, q}$, for $p$ or $q=\infty$, do not have unconditional bases.)

Thus any function (or distribution) $f$ which belongs to one of the previously mentioned function spaces can be written as

$$
f=\sum c_{j, k}^{(i)} \psi^{(i)}\left(2^{j} x-k\right)
$$

where

$$
c_{j, k}^{(i)}=2^{d j} \int f(x) \psi^{(i)}\left(2^{j} x-k\right) d x,
$$

and the series (2.1) converges to $f$ in the corresponding function space. (Note that in (2.1), wavelets are not normalized for the $L^{2}$ norm but for the $L^{\infty}$ norm, which will simplify some notations.)

In the following, we "forget" the index (i) of the wavelet, which is of no consequence. If $j \in \mathbb{Z}$ and $k=\left(k_{1}, \ldots, k_{d}\right) \in \mathbb{Z}^{d}$, we associate to the couple $(j, k)$ the dyadic cube

$$
\lambda=\left[\frac{k_{1}}{2^{j}}, \frac{k_{1}+1}{2^{j}}\right] \times \cdots \times\left[\frac{k_{d}}{2^{j}}, \frac{k_{d}+1}{2^{j}}\right] .
$$

We will use the notations $c_{j, k}$ or $c_{\lambda}$ indifferently for wavelet coefficients. We will assume in the following that the wavelet basis is $N$-smooth with $N \geq s+1$. Meyer proved that, if $s \in \mathbb{R}$ and $p>1$, then the Sobolev spaces have the following characterization in terms of wavelet coefficients (see [18, Chapter 6, Propositions 1 and 3]):

$$
f \in L^{p, s}\left(\mathbb{R}^{d}\right) \Longleftrightarrow\left\|\left(\sum_{j<0} \sum_{k \in \mathbb{Z}^{d}}\left|c_{\lambda}\right|^{2} \chi_{\lambda}(x)\right)^{1 / 2}\right\|_{L^{p}}+\left\|\left(\sum_{j \geq 0} \sum_{k \in \mathbb{Z}^{d}}\left|c_{\lambda}\right|^{2} 4^{j s} \chi_{\lambda}(x)\right)^{1 / 2}\right\|_{L^{p}}<\infty,
$$

where $\chi_{\lambda}(x)$ denotes the characteristic function of the dyadic cube $\lambda$. Homogeneous Besov spaces, which will also be considered, are characterized by the following condition, valid for $p, q \in(0,+\infty)$ and $s \in \mathbb{R}$,

$$
f \in B_{p}^{s, q}\left(\mathbb{R}^{d}\right) \Longleftrightarrow \sum_{j}\left(\sum_{k \in \mathbb{Z}^{d}}\left|c_{j, k}\right|^{p} 2^{(s p-d) j}\right)^{q / p}<\infty,
$$

see [18, Chapter 6] for $p \geq 1$ and [7, Chapter 7] for the general case with the usual extension if $p$ or $q$ is infinite; in particular, if $q=\infty$, this condition becomes

$$
\exists c \geq 0, \forall j \in \mathbb{Z}, \quad \sum_{k \in \mathbb{Z}^{d}}\left|c_{j, k}\right|^{p} 2^{(s p-d) j} \leq c .
$$

Note that, though $B_{p}^{s, q}$ does not have unconditional bases if $p$ or $q$ is infinite, nonetheless (2.5) or (2.6) still characterizes $B_{p}^{s, q}$ in this case. The only difference is that the finite sums $\sum c_{\lambda} \psi_{\lambda}$ do not converge strongly to $f$ in the space $B_{p}^{s, q}$ but in the weak* sense, that is,

$$
\forall g \in B_{p^{*}}^{-s, q^{*}}, \quad\left\langle f-\sum c_{\lambda} \psi_{\lambda} \mid g\right\rangle \longrightarrow 0
$$


We will not actually use the full unconditionality property in the following but rather the equivalent norms supplied by the wavelet characterizations, so that the conclusions of Theorem 2.1 are valid also for $p$ or $q$ infinite.

If $p \in(0,1)$, Besov spaces are no more Banach spaces but nonetheless are complete metric vector spaces. Note that, if $p \geq 1$, then Besov spaces are closely related to Sobolev spaces since the following embeddings hold:

$$
\forall p \geq 1, \forall s \in \mathbb{R}, \quad B_{p}^{s, 1} \hookrightarrow L^{p, s} \hookrightarrow B_{p}^{s, \infty}
$$

see [21, Chapter 4]. It follows that functions of $B_{p}^{s, q}$ notably differ from functions of $L^{p, s}$ only in the case $p<1$. However, the Sobolev embeddings still hold: for any $p, q>0$, if $s>d / p$, then $B_{p}^{s, q} \hookrightarrow C^{s-d / p}$ and the embedding is optimal in the same sense as in the Sobolev case, see [24, Chapter 11]. Thus, the same problem concerning the genericity of functions of $B_{p}^{s, q}$ having the worst possible Hölder exponent (i.e., $s-d / p$ ) at a given point $x_{0}$ can be raised. We will show that the following result, similar to Theorem 1.4, holds.

Theorem 2.1. Let $p, q \in(0,+\infty]$ and $s>d / p$; let $x_{0}$ be a given point of $\mathbb{R}^{d}$. The functions of $B_{p}^{s, q}$ having the pointwise Hölder exponent

$$
h_{f}\left(x_{0}\right)=s-\frac{d}{p}
$$

form an HP-residual set.

The following proposition (see $[10,11,12]$ ) will be used as a simple criterion for Hölder regularity or irregularity.

Proposition 2.2. Let $\alpha>0$ be given. There exists a constant $D$ which depends only on $\alpha$ and on the wavelet basis chosen such that, if $f$ is $(C, \alpha)$-smooth at $x_{0}$, then

$$
\exists J_{0}>0, \forall j \geq J_{0}, \forall k, \quad\left|c_{j, k}\right| \leq C \cdot D \cdot 2^{-\alpha j}\left(1+\left|2^{j} x_{0}-k\right|\right)^{\alpha} .
$$

Conversely, if

$$
\exists \alpha^{\prime}<\alpha, \exists J_{0}>0, \forall j \geq J_{0}, \forall k, \quad\left|c_{j, k}\right| \leq C \cdot D \cdot 2^{-\alpha j}\left(1+\left|2^{j} x_{0}-k\right|\right)^{\alpha^{\prime}},
$$

then $f \in C^{\alpha}\left(x_{0}\right)$.

We will use a weaker form of the first statement of Proposition 2.2 in the following section. If $x=\left(x_{1}, \ldots, x_{d}\right) \in \mathbb{R}^{d}$, let $[x]=\left(\left[x_{1}\right], \ldots,\left[x_{d}\right]\right)$; then, if $x_{0}$ is a given point of $\mathbb{R}^{d}$, let $k_{j}\left(=k_{j}\left(x_{0}\right)\right)=\left[2^{j} x_{0}\right]$; it follows from $(2.10)$ that

$$
\exists J_{0}>0, \forall j \geq J_{0} \quad\left|c_{j, k_{j}}\right| \leq C \cdot D \cdot 2^{-\alpha j} .
$$

The second part of the proposition will be used in the appendix. 


\section{Proof of Theorems 1.4 and 2.1}

In the following, we suppose that a (smooth enough) wavelet basis has been fixed once and for all. Since the notion of HP-small set does not depend on the choice of an equivalent norm (with a possible change of the constant of porosity $c$ ), the Sobolev and Besov norms (or quasinorms when $p, q<1$ ) used in the following are the ones which are implicitly defined by (2.4) or (2.5). We first prove the result in Besov spaces since they have a simpler wavelet characterization than Sobolev spaces. We will consider the Sobolev case afterwards.

Let $p, q>0, s$ be such that $s>d / p$; for a given $J_{0}>0$ and $H>s-d / p$, let

$$
A\left(J_{0}, H\right)=\left\{f \in B_{q}^{p, s} ; \forall j \geq J_{0}\left|c_{j, k_{j}}\right| \leq 2^{-H j}\right\} .
$$

Because of (2.12), the set of functions with a pointwise Hölder exponent larger than $s-$ $d / p$ at $x_{0}$ is contained in

$$
\bigcup_{J_{0} \in \mathbb{N}, H>s-d / p} A\left(J_{0}, H\right),
$$

and this union can be written as a countable union. We will actually prove that $A\left(J_{0}, H\right)$ is $\mathrm{HP}_{(1 / 4)}$. Let $c^{\prime} \in(0,1 / 4)$ and $r>0$ be fixed. We define $K$ as the smallest integer such that

$$
K>J_{0}, \quad 2^{(H-s+d / p)(K-1)} \geq \frac{4}{r} .
$$

For any $i \in \mathbb{N}$, let $y_{i}$ be the function defined by its wavelet coefficients (denoted by $c_{j, k}^{i}$ ) as follows:

$$
\text { if } k=k_{j}, j=i, \quad \text { then } c_{j, k}^{i}=r 2^{-(s-d / p) j} \text { else } c_{j, k}^{i}=0 .
$$

Each $y_{i}$ has only one coefficient different from zero and $\left\|y_{i}\right\|_{B_{p}^{s, q}}=r$. Suppose that there exists a function $f \in B_{p}^{s, q}$ such that

$$
\operatorname{card}\left\{i:\left(f+B\left(y_{i}, c^{\prime} r\right)\right) \cap A\left(J_{0}, H\right) \neq \varnothing\right\}>K \text {. }
$$

Thus there exist functions $f_{i_{1}}, \ldots, f_{i_{K}}$ such that, for all $l=1, \ldots, K$,

$$
\begin{gathered}
f-f_{i_{l}} \in B\left(y_{i_{l}}, c^{\prime} r\right), \\
f_{i_{l}} \in A\left(J_{0}, H\right) .
\end{gathered}
$$

In the following, we will denote by $f_{j, k}^{i_{l}}$ the wavelet coefficient of the function $f_{i_{l}}$. Since there are $K$ distinct functions $f_{i_{l}}$, at least two of them have indexes $i_{l}$ and $i_{m}$ which are not smaller than $K-1$. We can suppose that $i_{l}>i_{m}$. We now consider the wavelet coefficients corresponding to indexes $\left(j, k_{j}\right)$ with $j=i_{l}$ or $j=i_{m}$. It follows from (3.7) that $f-f_{i_{l}}-$ $y_{i_{l}} \in B\left(0, c^{\prime} r\right)$ and $f-f_{i_{m}}-y_{i_{m}} \in B\left(0, c^{\prime} r\right)$, hence

$$
\begin{aligned}
& \left|f_{i_{l}, k_{i_{l}}}^{i_{l}}\right| \leq 2^{-H i_{l}}, \\
& \left|f_{i_{l}, k_{i_{l}}}^{i_{m}}\right| \leq 2^{-H i_{l}},
\end{aligned}
$$


and it follows from (3.6) that

$$
\left\|\left(f_{i_{l}}-f_{i_{m}}\right)-\left(y_{i_{l}}-y_{i_{m}}\right)\right\| \leq 2 c^{\prime} r
$$

Using the wavelet characterization of the Besov norm, we obtain that

$$
\forall j, \quad\left|f_{j, k_{j}}^{i_{l}}-f_{j, k_{j}}^{i_{m}}-\left(c_{j, k_{j}}^{i_{l}}-c_{j, k_{j}}^{i_{m}}\right)\right| \leq 2 c^{\prime} r 2^{-(s-d / p) j} .
$$

Pick now $j=i_{l}$; using (3.4), (3.10) becomes

$$
\left|f_{i_{l}, k_{i_{l}}}^{i_{l}}-f_{i_{l}, k_{i_{l}}}^{i_{m}}-r 2^{-(s-d / p) i_{l}}\right| \leq 2 c^{\prime} r 2^{-(s-d / p) i_{l}}
$$

But (3.8) implies that

$$
\left|f_{i_{l}, k_{i_{l}}}^{i_{l}}-f_{i_{l}, k_{i_{l}}}^{i_{m}}\right| \leq 2.2^{-H i_{l}}
$$

Since $i_{l} \geq K-1$ and $H-s-d / p>0$, using (3.3), it follows that

$$
r 2^{-(s-d / p) i_{l}} \geq 2\left|f_{i_{l}, k_{i_{l}}}^{i_{l}}-f_{i_{l}, k_{i_{l}}}^{i_{m}}\right|
$$

so that

$$
\left|f_{i_{l}, k_{i_{l}}}^{i_{l}}-f_{i_{l}, k_{i_{l}}}^{i_{m}}-r 2^{-(s-d / p) i_{l}}\right| \geq \frac{r}{2} 2^{-(s-d / p) i_{l}}
$$

This is incompatible with (3.11), and Theorem 2.1 is proved.

The proof in the case of $L^{p, s}$ is similar; indeed we can pick the same functions $y_{i}$ which, because of the particular wavelet norm (2.4) that we chose for $L^{p, s}$, still satisfy $\left\|y_{i}\right\|_{L^{p, s}}=r$; (3.8) still holds and (3.10) also holds because it only involves the wavelet norms that we picked. The end of the proof runs the same.

\section{The Gaussian null setting}

The setting supplied by HP-residual results is fitted to obtain the conclusion of Theorem 1.4. However, one might wonder if there are other notions of genericity which also imply this conclusion. Such a notion, which has been used in several occurrences, was introduced by Phelps in 1978, see [22]. Recall that a Borel probability measure $\mu$ on a Banach space $E$ is a nondegenerate Gaussian measure if for every $g \in E^{*} \backslash\{0\}$, the measure $\mu \circ g^{-1}$ is a Gaussian measure on $\mathbb{R}$ which is not a Dirac mass.

Definition 4.1. A Borel subset $B$ of a separable Banach space $E$ is a Gaussian null set if, for every nondegenerate Gaussian measure $\mu$ on $E, \mu(B)=0$.

This notion coincides with Aronszajn null sets, as proved by Csörnyei in [4]. A Gaussian null set is necessarily Haar null. The following property illustrates the fact that Gaussian genericity is a very strong notion of genericity: in infinite-dimensional Banach spaces, there exist compact sets which are not Gaussian null (whereas they are always HP-small). 
We now prove that the conclusion of Theorem 1.4 cannot hold in this too strong setting. We only consider the case of $L^{p, s}$ with $p>1$ and $s>d / p$. Let $\chi_{j, k}$ be independent and identically distributed standard Gaussians, let $A_{j}=2^{-2^{|j|}}$, and consider the random function

$$
X(x)=\sum_{j, k} \chi_{j, k} A_{j} 2^{-|k|} \psi_{j, k}(x)
$$

where $\psi_{j, k}(x)=\Psi\left(2^{j} x-k\right)$, and the wavelet $\Psi$ is compactly supported. Let $N$ be an integer larger than $s$; we assume that $\Psi \in C^{N}\left(\mathbb{R}^{d}\right)$. The trivial bound

$$
\mathbb{P}\left(\left|\chi_{j, k}\right| \geq(1+|j|)(1+|k|)\right) \leq e^{-|j|} e^{-|k|}
$$

implies that, using the Borel-Cantelli lemma, almost surely, for all but finitely many pairs $(j, k)$, the $\chi_{j, k}$ satisfy

$$
\left|\chi_{j, k}\right| \leq(1+|j|)(1+|k|) \text {. }
$$

We check that the sample paths of $X(x)$ almost surely belong to $C^{N}\left(\mathbb{R}^{d}\right)$ and their partial derivatives up to order $N$ have fast decay. Indeed, if (4.3) holds, then (up to a finite linear combination of the wavelets which brings a compactly supported contribution in $C^{N}$ ), $X(x)$ can be bounded as follows.

For each $j$, there exist at most $C_{1}$ wavelets whose support includes $x$. Thus

$$
|X(x)| \leq \sum_{j} \sum_{k} A_{j} 2^{-|k|} C_{1} \leq C \cdot C_{1}
$$

Assume now that $|x| \geq 10$. The wavelets which bring a contribution to (4.1) satisfy $\mid 2^{j} x-$ $k \mid \leq D$, so that $|k| \geq\left|2^{j} x\right|-D$. Thus

$$
\sum_{j} \sum_{k} A_{j} 2^{-|k|}\left|\psi\left(2^{j} x-k\right)\right| \leq \sum_{j} A_{j} 2^{-\left|2^{j} x\right|} 2^{D} C_{1} \leq c 2^{-\sqrt{|x|}} .
$$

The estimates for the partial derivatives up to order $N$ are similar. Therefore the sample paths of $X$ are almost surely in $L^{p, s}$, and $X$ defines a measure on $L^{p, s}$ which will be denoted by $\mu$.

In order to check that this measure is a nondegenerate Gaussian measure, we first recall some additional properties of wavelet expansions in Sobolev spaces. The dual space of $L^{p, s}$ is $L^{p^{*},-s}$, see [18, Chapter 6].

Lemma 4.2. If $f=\sum f_{j, k} \psi_{j, k}$ belongs to $L^{p, s}$, and $g=\sum g_{j, k} \psi_{j, k}$ belongs to $L^{p^{*},-s}$, then

$$
\langle f \mid g\rangle_{L^{p, s}, L^{p^{*},-s}}=\sum_{j, k} f_{j, k} g_{j, k}
$$

Though this lemma is implicitly contained in several textbooks on wavelets (for instance, it underlies the wavelet characterization of the Sobolev spaces $L^{p, s}$ when $s<0$ in [18, Chapter 6]), we sketch its proof for the reader's convenience. 
Assume that $f$ and $g$ are finite linear combinations of wavelets. Then, by definition of $L^{p, s}$ and $L^{p^{*},-s}$,

$$
\begin{aligned}
\langle f \mid g\rangle_{L^{p, s}, L^{p^{*},-s}} & =\left\langle(\operatorname{Id}-\Delta)^{s / 2} f \mid(\operatorname{Id}-\Delta)^{-s / 2} g\right\rangle_{L^{p}, L^{p^{*}}} \\
& =\sum_{j, k} \sum_{j^{\prime}, k^{\prime}} f_{j, k} g_{j^{\prime}, k^{\prime}}\left\langle(\operatorname{Id}-\Delta)^{s / 2} \psi_{j, k} \mid(\operatorname{Id}-\Delta)^{-s / 2} \psi_{j^{\prime}, k^{\prime}}\right\rangle \\
& =\sum_{j, k} \sum_{j^{\prime}, k^{\prime}} f_{j, k} g_{j^{\prime}, k^{\prime}}\left\langle\psi_{j, k} \mid \psi_{j^{\prime}, k^{\prime}}\right\rangle \\
& =\sum_{j, k} f_{j, k} g_{j, k} .
\end{aligned}
$$

By a standard density argument, this equality holds for any couple $(f, g) \in L^{p, s} \times L^{p^{*},-s}$, in which case the series in the right-hand side of (4.6) converges absolutely (because of the unconditionality of the wavelet basis).

We can now check that $\mu$ is nondegenerate. Indeed, $\mu \circ g^{-1}$ is the Gaussian random variable $\sum \chi_{j, k} A_{j} 2^{-|k|} g_{j, k}$, which has the variance

$$
\sum_{j, k} A_{j}^{2} 2^{-2|k|}\left|g_{j, k}\right|^{2}
$$

and therefore is nondegenerate if $g \neq 0$.

We finally check that $\mu$ is indeed a Borel measure. Let $E$ be the Hilbert space defined by

$$
f=\sum_{j, k} c_{j, k} \Psi_{j, k} \in E \Longleftrightarrow \sum_{j, k}\left|c_{j, k}\right|^{2} 2^{2^{|j|}} 2^{|k|}<\infty
$$

The sample paths of the process $X$ clearly belong to $E$ so that $X$ defines a probability measure on $E$.

To check that $\mu$ is a Borel measure on $E$, we use the following lemma from $[16$, Section $8]$.

Leмma 4.3. Let E be a separable metrizable locally convex space. Then the $\sigma$-algebra generated by

$$
\left\{x \in E\left(f_{1}(x), \ldots, f_{n}(x)\right) \in A\right\}, \quad A \in \mathscr{B}^{n}, f_{j} \in E^{*},
$$

coincides with the Borel algebra.

Therefore, in order to check that $X$ defines a Borel measure, we only have to check that the sets $\left\{x \in E\left(f_{1}(x), \ldots, f_{n}(x)\right) \in A\right\}$ are measurable for the measure induced by $X$.

But if $f_{i}=\sum_{j, k} c_{j, k}^{i} \Psi_{j, k}$ are elements of $E^{*}=E$, then for $x=\sum_{j, k} d_{j, k} \Psi_{j, k}$, the event $\left(f_{1}(x), \ldots, f_{n}(x)\right) \in A$ is given by

$$
\left(\sum_{j, k} c_{j, k}^{1} d_{j, k} 2^{2^{|j|}} 2^{|k|}, \ldots, \sum_{j, k} c_{j, k}^{n} d_{j, k} 2^{2^{|j|}} 2^{|k|}\right) \in A
$$


But the probability of this event under $\mu$ is given by the probability of

$$
\left(\sum_{j, k} c_{j, k}^{1} \chi_{j, k}, \ldots, \sum_{j, k} c_{j, k}^{n} \chi_{j, k}\right) \in A
$$

As $\chi_{j, k}$ are independant, $\left(\sum_{j, k} c_{j, k}^{1} \chi_{j, k}, \ldots, \sum_{j, k} c_{j, k}^{n} \chi_{j, k}\right)$ is a Gaussian vector on $\mathbb{R}^{n}$, thus $\left\{x \in E\left(f_{1}(x), \ldots, f_{n}(x)\right) \in A\right\}$ is measurable. And $\mu$ is a Borel measure on $E$.

Moreover, the Hilbert space $E$ is clearly embedded in $L^{p, s}$, thus $\mu$ defines a Borel measure on $L^{p, s}$.

Since the sample paths of $X(x)$ almost surely are $C^{N}$ with derivatives up to order $N$ with fast decay, the set of functions $f$ satisfying $h_{f}\left(x_{0}\right)=s-d / p$ has $\mu$-measure 0 . It follows that the complement of this set is not Gaussian null.

Remark 4.4. There is no $H$ such that $h_{f}\left(x_{0}\right)=H$ for every $f$ outside of a Gaussian null subset of $L^{p, s}$. Indeed, then this would be true for $f$ in a prevalent set, hence the only possible value for $H$ is $H=s-d / p$ by Theorem 1.4. However, this contradicts the result of this section.

\section{Appendix}

\section{Results valid for all functions}

For the reader's convenience, we recall or prove several pointwise regularity properties which are valid for all functions. First, note that, if $s>d / p$, then a simple example of a function $f \in L^{p, s} \cap B_{p}^{s, q}$ satisfying property ( $\mathscr{H}$ ), that is, such that $h_{f}\left(x_{0}\right)=s-d / p$, is supplied by any function such that if $\left|x-x_{0}\right| \leq 1 / 2$, then

$$
f(x)=\left|x-x_{0}\right|^{s-d / p}\left|\log \left(\left|x-x_{0}\right|\right)\right|^{-a}, \quad \text { with } a>\frac{1}{p}\left(a>\frac{1}{p}+\frac{1}{q} \text { in } B_{p}^{s, q}\right) .
$$

By a simple superposition argument, one can deduce a function $f$ which has Hölder exponent $s-d / p$ on a countable dense set of points. However, one cannot increase much the size of the set of discontinuities; indeed, the set of points $x_{0}$ satisfying $h_{f}\left(x_{0}\right)=s-d / p$ has to be of Hausdorff dimension 0 as a consequence of the following general results of [11, Proposition 4.1].

Proposition A.1. Let $s>d / p$ and let $f$ be an arbitrary function in $B_{p}^{s, \infty}\left(\mathbb{R}^{d}\right)$; denote by $d_{f}(H)$ the Hausdorff dimension of the set of points $x$, where $h_{f}(x)=H$. Then $d_{f}(H) \leq$ $d-(s-H) p$.

The result follows by applying this proposition with $H=s-d / p$ and keeping in mind that the spaces $B_{p}^{s, q}$ and $L^{p, s}$ are included in $B_{p}^{s, \infty}$.

The almost everywhere regularity of a function of $L^{p, s}$ or $B_{p}^{s, q}$ is much better, as a consequence of the following proposition.

Proposition A.2. Let $s>d / p$ and $f \in B_{p}^{s, p}\left(\mathbb{R}^{d}\right)$; then, for almost every $x_{0}, f \in C^{s}\left(x_{0}\right)$. 
Proof. Let $\varphi(x)=(1+|x|)^{-a}$ with $d<a<s p$, so that $\varphi \in L^{1}$. Let

$$
g(x)=\sum_{\lambda \in \Lambda}\left|c_{\lambda}\right|^{p} 2^{s p j} \varphi\left(2^{j} x-k\right)
$$

where the $c_{\lambda}$ are the wavelet coefficients of $f$. Since (A.2) has only nonnegative terms,

$$
\|g\|_{L^{1}}=C \sum_{\lambda}\left|c_{\lambda}\right|^{p} 2^{(s p-d) j}
$$

which is finite, since $f \in B_{p}^{s, p}$. (Note that $C=\|\varphi\|_{L^{1}}$, which explains why we pick $a>d$.) It follows that (A.2) is almost everywhere finite so that, for almost every $x$, we have

$$
K:=\sum_{\lambda \in \Lambda}\left|c_{\lambda}\right|^{p} \frac{2^{s p j}}{\left(1+\left|2^{j} x-k\right|\right)^{a}}<\infty .
$$

In particular,

$$
\forall \lambda, \quad\left|c_{\lambda}\right| \leq K^{1 / p} 2^{-s j}\left(1+\left|2^{j} x-k\right|\right)^{a / p}
$$

Since $a / p<s$, it follows from Proposition 2.2 that $f \in C^{s}(x)$.

Corollary A.3. If $f$ belongs to $L^{p, s}$ or $B_{p}^{s, q}$ with $s>d / p$, then for almost every $x, h_{f}\left(x_{0}\right) \geq s$.

Indeed, we pick $s^{\prime}$ such that $s>s^{\prime}>d / p$; since $f \in B_{p}^{s^{\prime}, p}$, it follows that a.e. $f \in C^{s^{\prime}}(x)$. Since $s^{\prime}$ can be chosen arbitrarily close to $s$, the corollary follows.

\section{Acknowledgments}

The authors thank the referee and Simeon Reich for suggesting many substantial improvements upon a first version of this paper. Stéphane Jaffard is supported by the Institut Universitaire de France.

\section{References}

[1] H. Auerbach and S. Banach, Über die Höldersche Bedingung, Studia Math. 3 (1931), 180-184 (German).

[2] S. Banach, Über die Bairesche Kategorie gewisser Funktionenmengen, Studia Math. 3 (1931), 174-179 (German).

[3] J. P. R. Christensen, On sets of Haar measure zero in abelian Polish groups, Israel J. Math. 13 (1972), 255-260.

[4] M. Csörnyei, Aronszajn null and Gaussian null sets coincide, Israel J. Math. 111 (1999), 191201.

[5] I. Daubechies, Ten Lectures on Wavelets, CBMS-NSF Regional Conference Series in Applied Mathematics, vol. 61, SIAM, Pennsylvania, 1992.

[6] A. Fraysse and S. Jaffard, How smooth is almost every function in a Sobolev space? to appear in Rev. Mat. Iberoamericana.

[7] M. Frazier, B. Jawerth, and G. Weiss, Littlewood-Paley Theory and the Study of Function Spaces, CBMS Regional Conference Series in Mathematics, vol. 79, American Mathematical Society, Rhode Island, 1991. 
[8] B. R. Hunt, The prevalence of continuous nowhere differentiable functions, Proc. Amer. Math. Soc. 122 (1994), no. 3, 711-717.

[9] B. R. Hunt, T. Sauer, and J. A. Yorke, Prevalence: a translation-invariant "almost every" on infinite-dimensional spaces, Bull. Amer. Math. Soc. (N.S.) 27 (1992), no. 2, 217-238.

[10] S. Jaffard, Exposants de Hölder en des points donnés et coefficients d'ondelettes [Holder exponents at given points and wavelet coefficients], C. R. Acad. Sci. Paris Sér. I Math. 308 (1989), no. 4, 79-81 (French).

[11] Multifractal formalism for functions. I. Results valid for all functions, SIAM J. Math. Anal. 28 (1997), no. 4, 944-970.

[12] _ Multifractal formalism for functions. II. Self-similar functions, SIAM J. Math. Anal. 28 (1997), no. 4, 971-998.

[13] On the Frisch-Parisi conjecture, J. Math. Pures Appl. (9) 79 (2000), no. 6, 525-552.

[14] S. Jaffard and Y. Meyer, On the pointwise regularity of functions in critical Besov spaces, J. Funct. Anal. 175 (2000), no. 2, 415-434.

[15] J. Kolár, Porous sets that are Haar null, and nowhere approximately differentiable functions, Proc. Amer. Math. Soc. 129 (2001), no. 5, 1403-1408.

[16] M. A. Lifshits, Gaussian Random Functions, Mathematics and Its Applications, vol. 322, Kluwer Academic, Dordrecht, 1995.

[17] S. Mazurkiewicz, Sur les fonctions non derivables, Studia Math. 3 (1931), 92-94 (French).

[18] Y. Meyer, Ondelettes et Opérateurs. I. [Wavelets and operators. I], Actualités Mathématiques, Hermann, Paris, 1990.

[19] - Ondelettes et Opérateurs. II. [Wavelets and operators. II], Actualités Mathématiques, Hermann, Paris, 1990.

[20] Y. Meyer and R. R. Coifman, Ondelettes et Opérateurs. III. [Wavelets and operators. III], Actualités Mathématiques, Hermann, Paris, 1991.

[21] J. Peetre, New Thoughts on Besov Spaces, Duke University Mathematics Series, no. 1, Mathematics Department, Duke University, North Carolina, 1976.

[22] R. R. Phelps, Gaussian null sets and differentiability of Lipschitz map on Banach spaces, Pacific J. Math. 77 (1978), no. 2, 523-531.

[23] D. Preiss and J. Tišer, Two unexpected examples concerning differentiability of Lipschitz functions on Banach spaces, Geometric Aspects of Functional Analysis (Israel, 1992-1994), Oper. Theory Adv. Appl., vol. 77, Birkhäuser, Basel, 1995, pp. 219-238.

[24] H. Triebel, The Structure of Functions, Monographs in Mathematics, vol. 97, Birkhäuser, Basel, 2001.

A. Fraysse: Laboratoire d'Analyse et de Mathématiques Appliquées, Université Paris XII Val de Marne, 61 avenue du Général de Gaulle, 94010 Créteil Cedex, France

E-mail address: fraysse@univ-paris12.fr

S. Jaffard: Laboratoire d'Analyse et de Mathématiques Appliquées, Université Paris XII Val de Marne, 61 avenue du Général de Gaulle, 94010 Créteil Cedex, France

E-mail address: jaffard@univ-paris12.fr 


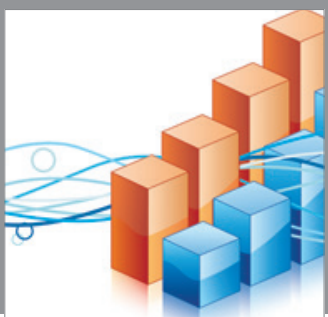

Advances in

Operations Research

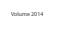

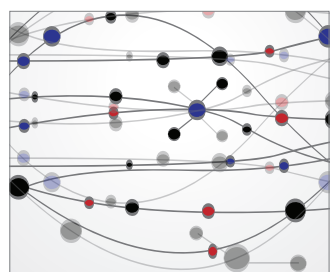

\section{The Scientific} World Journal
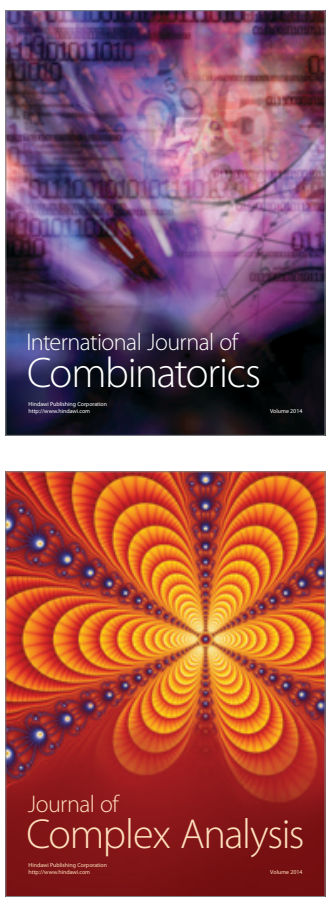

International Journal of

Mathematics and

Mathematical

Sciences
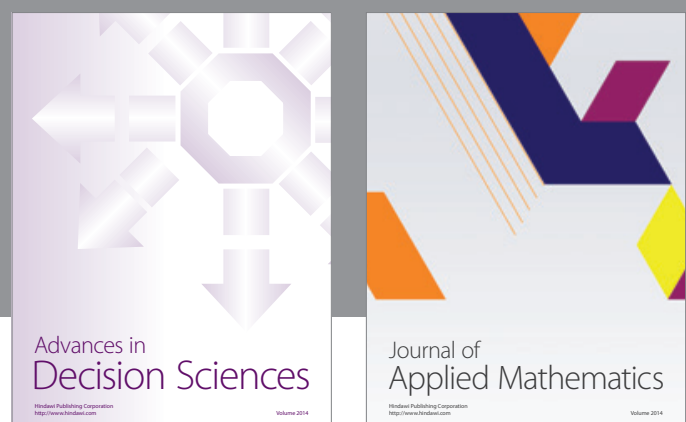

Journal of

Applied Mathematics
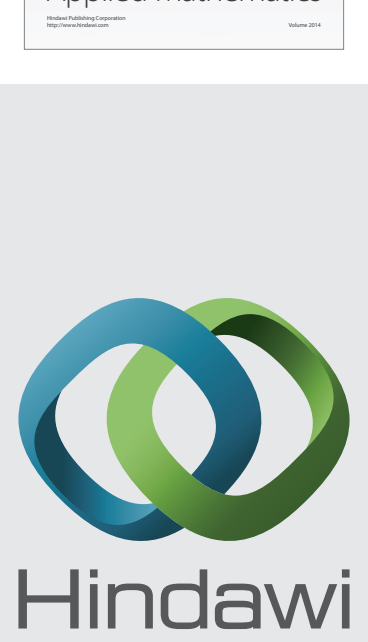

Submit your manuscripts at http://www.hindawi.com
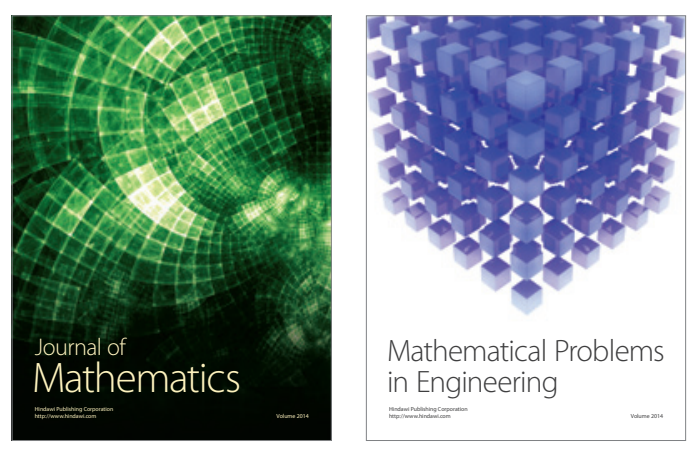

Mathematical Problems in Engineering
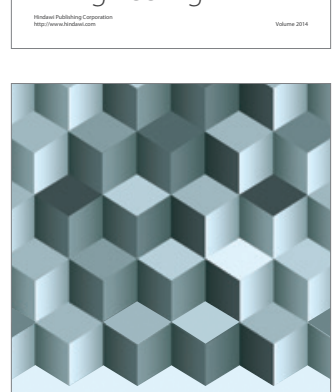

Journal of

Function Spaces
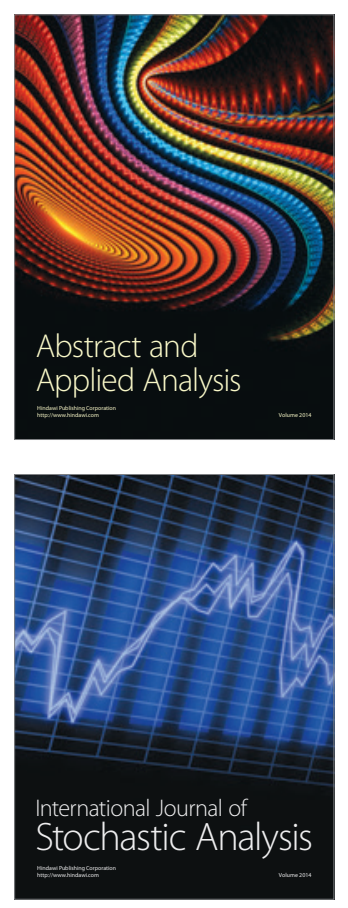

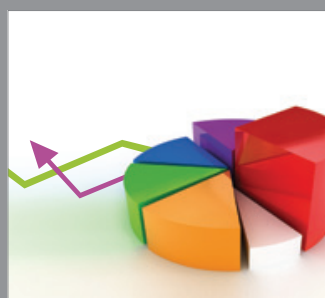

ournal of

Probability and Statistics

Promensencen
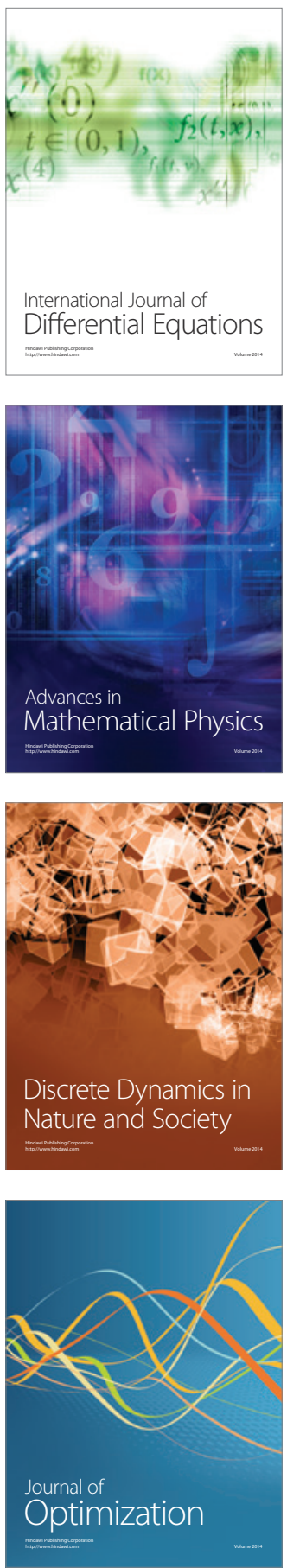